\title{
Synthesis of Multisubstituted Indenes via Iron-Catalyzed Domino Reaction of Benzylic Compounds and Alkynes
}

\author{
Yongxin Chen, ${ }^{\mathrm{a}, \mathrm{b}}$ Kangning Li, ${ }^{\mathrm{a}, \mathrm{b}}$ Xiang Liu, ${ }^{\mathrm{a}, \mathrm{b}}$ Jiaoyan Zhu, ${ }^{\mathrm{a}, \mathrm{b}}$ Baohua Chen*a,b \\ a State Key Laboratory of Applied Organic Chemistry, Lanzhou University, Lanzhou 730000, P. R. of China \\ ${ }^{b}$ Key Laboratory of Nonferrous Metal Chemistry and Resources Utilization of Gansu Province, Lanzhou 730000, P. R. of China \\ Fax +86(931)8912582; E-mail: chbh@1zu.edu.cn
}

Received: 18.10.2012; Accepted after revision: 07.11.2012

\begin{abstract}
A novel approach to synthesizing multisubstituted indenes by iron-catalyzed domino reaction of benzylic compounds and alkynes under mild conditions was developed. This system could be applied to various available substrates in a one-step synthetic procedure in moderate to good yields.
\end{abstract}

Key words: indenes, iron catalysis, domino reaction, one-step procedure, annulation

The indene core is one of the most important cyclic motifs that are very common in natural products and biologically active compounds. ${ }^{1}$ In addition, it has tremendous applications in various research fields including material chemistry, analytical chemistry, and synthetic organic chemistry. ${ }^{2}$ Great progress has been made to successfully construct this motif, but traditional methods required expensive and difficult-to-prepare catalysts/reagents, tedious reaction procedures, moreover, it is difficult to directly introduce multifunctional groups into the indene core. ${ }^{3}$ Recently, several new approaches ${ }^{4}$ to generating indenes from the reaction between carbon-carbon triple bonds and benzyl cation intermediates that are obtained by the use of leaving groups have been developed (Scheme 1, A).

In recent years, the direct functionalization of benzylic $\mathrm{C}\left(\mathrm{sp}^{3}\right)-\mathrm{H}$ bonds has attracted extensive attention in organic synthesis. ${ }^{5-8}$ For example, Shi and others ${ }^{5}$ have reported the cross-dehydrogenative coupling (CDC) between benzylic $\mathrm{C}-\mathrm{H}$ bonds and $\mathrm{sp}^{3}, \mathrm{sp}^{2}$, or sp $\mathrm{C}-\mathrm{H}$ bonds. Direct benzylic $\mathrm{C}-\mathrm{H}$ amination through various catalytic systems have also been developed. ${ }^{6}$ Additionally, the Jiao group $^{7}$ demonstrated an iron-catalyzed transformation of benzylic compounds into corresponding amide through $\mathrm{C}-\mathrm{H}$ and $\mathrm{C}-\mathrm{C}$ bond cleavage. Among these reactions, iron is a particularly attractive catalyst because of its sustainability, easy availability, low price, and environmentally friendly characteristics. ${ }^{9}$ Despite the significant progress that has been achieved in this area, the cyclization reaction is still a relatively unexplored field. Owing to the importance of indene derivatives and the lack of successful direct annulations from benzylic compounds without leaving groups, we initially envisioned a novel and effi-

SYNLETT 2013, 24, 0130-0134

Advanced online publication: 06.12.2012

DOI: 10.1055/s-0032-1317705; Art ID: ST-2012-W0900-L

(C) Georg Thieme Verlag Stuttgart · New York cient way of synthesizing multisubstituted indenes by in situ generated benzyl bromides as benzyl cation precursors in an iron-catalyzed, one-step synthetic procedure (Scheme 1, B).

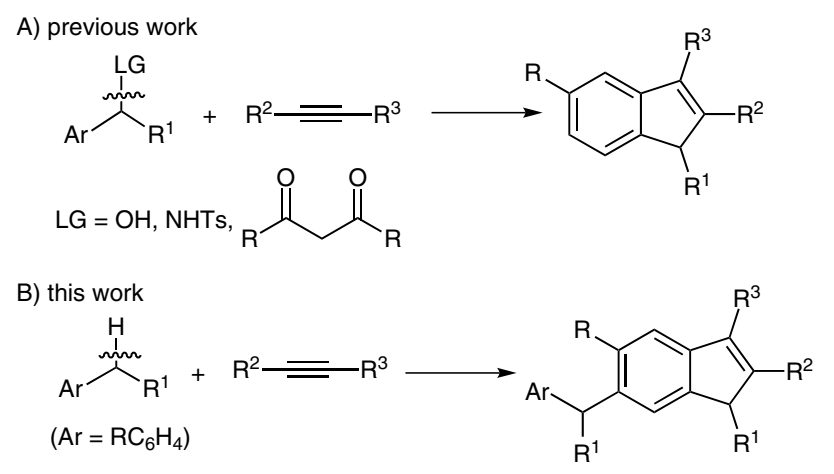

Scheme 1 Synthesis of indene derivatives

At the outset of our study, diphenylacetylene (1a) and diphenylmethane (2a) were chosen as model substrates to optimize the reaction conditions (Table 1). Compounds 3aa and 4aa were obtained in $10 \%$ and $56 \%$ yields, respectively, with $\mathrm{FeCl}_{2}(10 \mathrm{~mol} \%)$ as the catalyst and DDQ (2.0 equiv) as the oxidant in DCE at $80{ }^{\circ} \mathrm{C}$ (Table 1, entry1). The yield of 3aa increased to $66 \%$ when NBS was used as the oxidant, while other oxidants such as NCS, TBHP, and $\mathrm{Cu}(\mathrm{OAc})_{2}$ showed no effect on the promotion of this domino reaction (Table 1, entries 2-5). Subsequently, a series of solvents (toluene, acetonitrile, nitromethane, and tetrahydrofuran) were also evaluated but they showed disappointing results (Table 1, entries 69). Furthermore, we investigated the reaction using other iron, copper, and zinc salts as catalyst, but no better results were obtained (Table 1, entries 10-14). It is noteworthy that the amount of NBS had a significant effect on this reaction. Both 1.5 equivalents and 3.0 equivalents of NBS offered the desired product 3aa in a low yield; 2.2 equivalents of NBS were the most effective and resulted in $75 \%$ yield (Table 1, entries 15-17). In addition, the yield was found to decrease with the temperature changed to $60^{\circ} \mathrm{C}$ or $120^{\circ} \mathrm{C}$ (Table 1, entries 18 and 19 ), and only a trace amount of product was observed in the absence of NBS or $\mathrm{FeCl}_{2}$.

Under the optimized reaction conditions, a series of substituted substrates was investigated to establish the scope and limitations of this process (Table 2). The results 
Table 1 Optimization of the Reaction Conditions ${ }^{\mathrm{a}}$

\begin{tabular}{|c|c|c|c|c|c|c|}
\hline $1 \mathrm{a}$ & $2 a$ & & & 4aa & & \\
\hline Entry & Oxidant (equiv) & Catalyst & Solvent & Temp $\left({ }^{\circ} \mathrm{C}\right)$ & Yield of 3aa (\%) & Yield of 4aa (\%) \\
\hline 1 & DDQ (2.0) & $\mathrm{FeCl}_{2}$ & DCE & 80 & 10 & 56 \\
\hline 2 & NBS (2.0) & $\mathrm{FeCl}_{2}$ & DCE & 80 & 66 & 12 \\
\hline 3 & $\operatorname{NCS}(2.0)$ & $\mathrm{FeCl}_{2}$ & DCE & 80 & trace & 0 \\
\hline 4 & TBHP (2.0) & $\mathrm{FeCl}_{2}$ & DCE & 80 & trace & 0 \\
\hline 5 & $\mathrm{Cu}(\mathrm{OAc})_{2}(2.0)$ & $\mathrm{FeCl}_{2}$ & DCE & 80 & trace & 0 \\
\hline 6 & NBS (2.0) & $\mathrm{FeCl}_{2}$ & $\mathrm{PhMe}$ & 80 & 15 & 42 \\
\hline 7 & NBS (2.0) & $\mathrm{FeCl}_{2}$ & $\mathrm{MeCN}$ & 80 & 0 & 0 \\
\hline 8 & NBS (2.0) & $\mathrm{FeCl}_{2}$ & $\mathrm{MeNO}_{2}$ & 80 & 0 & 0 \\
\hline 9 & NBS (2.0) & $\mathrm{FeCl}_{2}$ & THF & 80 & 0 & 0 \\
\hline 10 & NBS (2.0) & $\mathrm{FeBr}_{2}$ & DCE & 80 & 43 & 0 \\
\hline 11 & NBS (2.0) & $\mathrm{FeCl}_{3}$ & DCE & 80 & 44 & 15 \\
\hline 12 & NBS (2.0) & $\mathrm{Fe}(\mathrm{acac})_{3}$ & DCE & 80 & trace & 20 \\
\hline 13 & NBS (2.0) & $\mathrm{CuCl}_{2}$ & DCE & 80 & trace & 0 \\
\hline 14 & NBS (2.0) & $\mathrm{ZnCl}_{2}$ & DCE & 80 & trace & 0 \\
\hline 15 & NBS (1.5) & $\mathrm{FeCl}_{2}$ & DCE & 80 & 56 & 20 \\
\hline 16 & NBS (2.2) & $\mathrm{FeCl}_{2}$ & DCE & 80 & 75 & 0 \\
\hline 17 & NBS (3.0) & $\mathrm{FeCl}_{2}$ & DCE & 80 & 47 & 0 \\
\hline 18 & NBS (2.2) & $\mathrm{FeCl}_{2}$ & DCE & 60 & 67 & 0 \\
\hline 19 & NBS (2.2) & $\mathrm{FeCl}_{2}$ & DCE & 120 & 60 & 0 \\
\hline
\end{tabular}

${ }^{\text {a }}$ Reaction conditions: 1a $(0.25 \mathrm{mmol}), \mathbf{2 a}(1.25 \mathrm{mmol})$, catalyst $(0.025 \mathrm{mmol})$, solvent $(2 \mathrm{~mL})$, under $\mathrm{N}_{2}, 12 \mathrm{~h}$.

showed that substrates with various weakly electron-donating and electron-withdrawing functional groups on either alkynes or benzylic compounds gave the corresponding indenes in moderate to good yields, and other strongly electron-donating and electron-withdrawing substituents gave no desired products probably because of strong electronic effect (Table 2, entries 7, 8, and 17). The corresponding product $3 d$ a was obtained in good yield when the substrate with an ortho-substituted aryl group was employed (Table 2, entry 4). Moreover, substrates with different substituents on $\mathrm{R}^{1}$ and $\mathrm{R}^{2}$ gave a mixture of regioisomers; due to electronic effects, the ratio of isomers varied from 3.7:1 to 1.3:1 (Table 2, entries 2, 4, 5; see also the Supporting Information). ${ }^{4 a, b}$ The aliphatic alkynes gave lower but still acceptable yields with perfect regioselectivity (Table 2 , entries 9 and 10). ${ }^{4 a, b}$ When unsymmetric diphenylmethanes were employed, exclusive regioselectivity of indenes were observed ex- cept for 3ab and 3ad (Table 2, entries 11, 13-19; see also the Supporting Information). Confirmed by ${ }^{1} \mathrm{H}$ NMR and ${ }^{13} \mathrm{C}$ NMR analyses of intermediate product 4ae, we assumed the favorable isomer would be 3ae. ${ }^{4 \mathrm{~b}}$ A low yield was obtained when the bis[4-(tert-butyl)phenyl]methane was used, probably because of the steric effects of the tertbutyl substituent (Table 2, entry 12). Besides diarylmethanes, simple benzylic substrates such as ethyl benzene also worked smoothly and gave a mixture of diastereomers (1:1 ratio) in 56\% yield (Table 2 , entry 16 ).

Interestingly, it was found that substrates with a para-methoxy group on the aryl ring could react smoothly by using DDQ as oxidant (Table 3, entry 1). Good yield was still achieved when the bis[4-(tert-butyl)phenyl]methane was used (Table 3 , entry 2 ).

To gain an insight into the mechanism of the above-mentioned process, the following control experiments were performed. As shown in Scheme 2, (bromometh- 
ylene)dibenzene A was obtained via reaction of diphenylmethane (2a) with NBS in DCE under optimized conditions (Scheme 2, A), and then diphenylacetylene (1a) and $\mathrm{FeCl}_{2}$ were added to the reaction mixture (Scheme 2, B), and 3aa was obtained in 74\% yield.

On the basis of the above results, a tentative reaction mechanism is illustrated in Scheme 2 (C). Treatment of diphenylmethane (2a) with NBS produces (bromomethylene)dibenzene $\mathbf{A}$. The reaction of iron salt on $\mathbf{A}$ can lead to benzylic cation, which regioselectively attacks $\mathbf{1 a}$ resulting in the formation of vinyl cation $\mathbf{C}$. Then $\mathbf{C}$ undergoes cyclization and subsequent aromatization to provide 4aa. ${ }^{4 a, b, 10}$ Finally, 4aa and a second $\mathbf{A}$ react to afford the desired product 3aa. ${ }^{11}$
In summary, we have developed a novel iron-catalyzed direct cyclization for the synthesis of multisubstituted indenes with benzylic compounds and alkynes in moderate to good yields. This system could be applied to various available substrates in a one-step synthetic procedure. We believe that this is one of the simplest and most straightforward methods available for the synthesis of indenes to date.

\section{Acknowledgment}

We are grateful to the project sponsored by the Project of National Science Foundation of P. R. of China (No. J11003307).

Supporting Information for this article is available online at http://www.thieme-connect.com/ejournals/toc/synlett.

Table 2 Synthesis of Indenes Derivatives from Alkynes and Benzylic Compounds ${ }^{\mathrm{a}}$
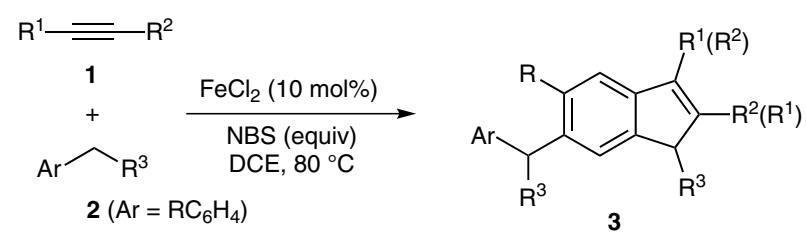

\begin{tabular}{|c|c|c|c|c|}
\hline Entry & $1 \mathrm{R}^{1}, \mathrm{R}^{2}$ & $2 \mathrm{Ar}, \mathrm{R}^{3}$ & NBS (equiv) & Yield of $\mathbf{3}(\%)$ \\
\hline 1 & 1a $\mathrm{Ph}, \mathrm{Ph}$ & 2a Ph, $\mathrm{Ph}$ & 2.2 & 3aa 75 \\
\hline 2 & 1b $\mathrm{Ph}, 4-\mathrm{MeC}_{6} \mathrm{H}_{4}$ & 2a $\mathrm{Ph}, \mathrm{Ph}$ & 1.9 & $\mathbf{3 b a} / \mathbf{3} \mathbf{b a}^{\prime}(1: 3.7) 72$ \\
\hline 3 & 1c $4-\mathrm{MeC}_{6} \mathrm{H}_{4}, 4-\mathrm{MeC}_{6} \mathrm{H}_{4}$ & 2a $\mathrm{Ph}, \mathrm{Ph}$ & 1.6 & 3ca 53 \\
\hline 4 & 1d $\mathrm{Ph}, 2-\mathrm{MeC}_{6} \mathrm{H}_{4}$ & 2a Ph, $\mathrm{Ph}$ & 1.8 & 3da/3da' (1:1.3) 69 \\
\hline 5 & 1e $\mathrm{Ph}, 4-\mathrm{ClC}_{6} \mathrm{H}_{4}$ & 2a Ph, $\mathrm{Ph}$ & 2.2 & 3ea/3ea' (1:1.3) 83 \\
\hline 6 & 1f $4-\mathrm{ClC}_{6} \mathrm{H}_{4}, 4-\mathrm{ClC}_{6} \mathrm{H}_{4}$ & 2a Ph, $\mathrm{Ph}$ & 2.5 & 3fa 74 \\
\hline 7 & $1 \mathrm{~g} \mathrm{Ph}, 4-\mathrm{O}_{2} \mathrm{NC}_{6} \mathrm{H}_{4}$ & 2a Ph, $\mathrm{Ph}$ & 2.2 & 3ga 0 \\
\hline 8 & 1h $\mathrm{Ph}, 4-\mathrm{MeOC}_{6} \mathrm{H}_{4}$ & 2a Ph, $\mathrm{Ph}$ & 2.2 & 3ha 0 \\
\hline 9 & 1i Ph, Bu & 2a $\mathrm{Ph}, \mathrm{Ph}$ & 3.0 & 3ia 55 \\
\hline 10 & $\mathbf{1 j} n-\operatorname{Pr}, n-\operatorname{Pr}$ & 2a $\mathrm{Ph}, \mathrm{Ph}$ & 2.2 & 3ja 42 \\
\hline 11 & 1a $\mathrm{Ph}, \mathrm{Ph}$ & 2b Ph, 4- $\mathrm{MeC}_{6} \mathrm{H}_{4}$ & 2.2 & 3ab $(\mathrm{Ar}=\mathrm{Ph}) / \mathbf{3} \mathbf{a} \mathbf{b}^{\prime}\left(\mathrm{Ar}=4-\mathrm{MeC}_{6} \mathrm{H}_{4}\right)(1: 1) 62$ \\
\hline 12 & 1a Ph, $\mathrm{Ph}$ & 2c $4-t-\mathrm{BuC}_{6} \mathrm{H}_{4}, 4-t-\mathrm{BuC}_{6} \mathrm{H}_{4}$ & 2.6 & $3 \mathbf{a c} 48^{\mathrm{c}}$ \\
\hline 13 & 1a Ph, Ph & 2d Ph, 4- $\mathrm{FC}_{6} \mathrm{H}_{4}$ & 2.6 & $\mathbf{3 a d}(\mathrm{Ar}=\mathrm{Ph}) / \mathbf{3} \mathbf{a d}^{\prime}\left(\mathrm{Ar}=4-\mathrm{FC}_{6} \mathrm{H}_{4}\right)(2: 1) 58$ \\
\hline 14 & 1a $\mathrm{Ph}, \mathrm{Ph}$ & 2e Ph, 4- $\mathrm{ClC}_{6} \mathrm{H}_{4}$ & 2.4 & 3ae 57 \\
\hline 15 & 1a $\mathrm{Ph}, \mathrm{Ph}$ & 2f $\mathrm{Ph}, 4-\mathrm{F}_{3} \mathrm{CC}_{6} \mathrm{H}_{4}$ & 3.0 & $3 a f 63^{c}$ \\
\hline 16 & 1a $\mathrm{Ph}, \mathrm{Ph}$ & $2 \mathrm{~g} \mathrm{Ph}, \mathrm{Me}$ & 2.2 & $\operatorname{3ag} 56^{\mathrm{d}}$ \\
\hline 17 & 1a $\mathrm{Ph}, \mathrm{Ph}$ & 2h Ph, 4- $\mathrm{MeOC}_{6} \mathrm{H}_{4}$ & 2.2 & 3ah 0 \\
\hline 18 & 1f $4-\mathrm{ClC}_{6} \mathrm{H}_{4}, 4-\mathrm{ClC}_{6} \mathrm{H}_{4}$ & 2e Ph, 4- $\mathrm{ClC}_{6} \mathrm{H}_{4}$ & 2.8 & 3fe 72 \\
\hline 19 & 1f $4-\mathrm{ClC}_{6} \mathrm{H}_{4}, 4-\mathrm{ClC}_{6} \mathrm{H}_{4}$ & 2f $\mathrm{Ph}, 4-\mathrm{F}_{3} \mathrm{CC}_{6} \mathrm{H}_{4}$ & 3.0 & $3 \mathbf{f f} 45^{\mathrm{b}}$ \\
\hline
\end{tabular}

${ }^{\mathrm{a}}$ Reaction conditions: $1(0.25 \mathrm{mmol}), \mathbf{2}(1.25 \mathrm{mmol}), \mathrm{FeCl}_{2}(0.025 \mathrm{mmol}), \mathrm{DCE}(2 \mathrm{~mL}), 80{ }^{\circ} \mathrm{C}, 10 \mathrm{~h}$, under $\mathrm{N}_{2}$.

${ }^{\mathrm{b}}$ At $100^{\circ} \mathrm{C}, 24 \mathrm{~h}$.

${ }^{c}$ At $100^{\circ} \mathrm{C}$.

${ }^{\mathrm{d}}$ At $60^{\circ} \mathrm{C}$. 
Table 3 Synthesis of Indene Derivatives Using DDQ as Oxidant ${ }^{\mathrm{a}}$

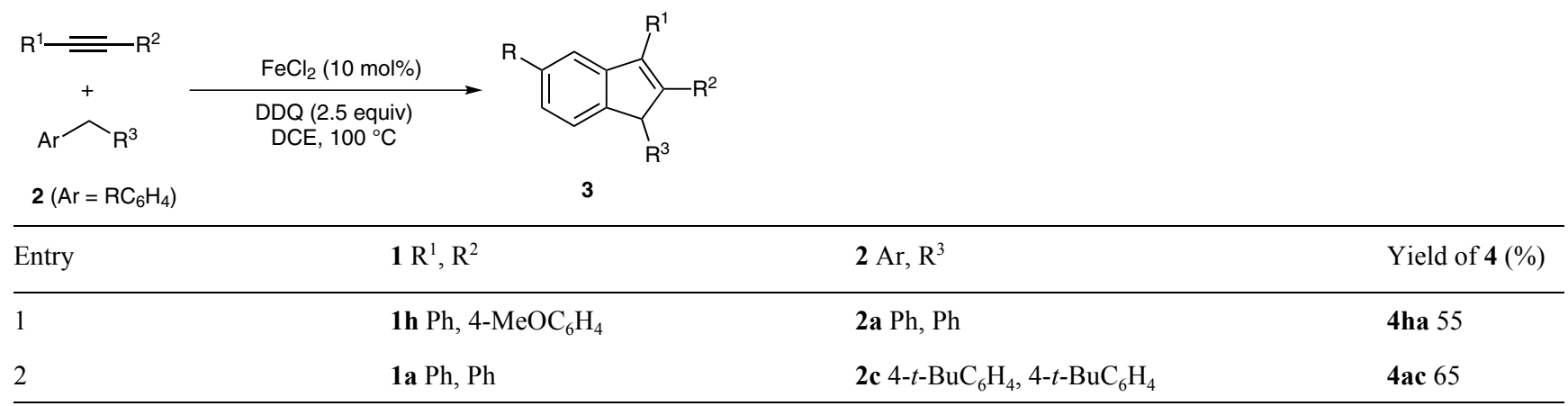

${ }^{\mathrm{a}}$ Reaction conditions: $1(0.25 \mathrm{mmol}), \mathbf{2}(1.25 \mathrm{mmol}), \mathrm{FeCl}_{2}(0.025 \mathrm{mmol})$, DDQ $(0.625 \mathrm{mmol}), \mathrm{DCE}(2 \mathrm{~mL}), 100{ }^{\circ} \mathrm{C}, 10 \mathrm{~h}$, under $\mathrm{N}_{2}$.

A)

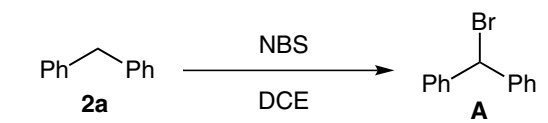

B)

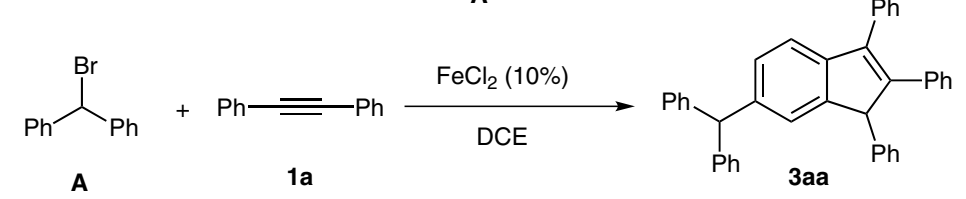

C)

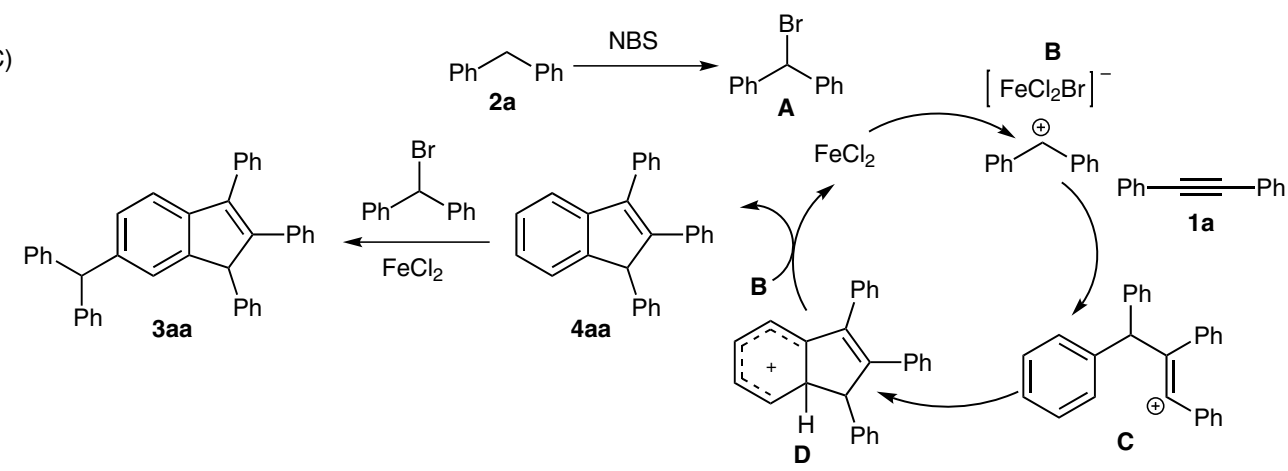

Scheme 2 A tentative reaction mechanism

\section{References and Notes}

(1) (a) Nugiel, D. A.; Etzkom, A.-M.; Vidwans, A.; Benfield, P. A.; Boisclair, M.; Burton, C. R.; Cox, S.; Czerniak, P. M.; Doleniak, D.; Seitz, S. P. J. Med. Chem. 2001, 44, 1334.

(b) Frédérick, R.; Dumont, W.; Ooms, F.; Aschenbach, L.; van der Schyf, C. J.; Castagnoli, N.; Wouters, J.; Krief, A. J. Med. Chem. 2006, 49, 3743. (c) Heintzelman, G. R.; Averill, K. M.; Dodd, J. H.; Demarest, K. T.; Tang, Y.; Jackson, P. F. WO 2003088963, 2003. (d) Safak, C.; Simsek, R.; Altas, Y.; Boydag, S.; Erol, K. Boll. Chim. Farm. 1997, 136, 665.

(2) (a) Anstead, G. M.; Wilson, S. R.; Katzenellenbogen, J. A. J. Med. Chem. 1989, 32, 2163. (b) Wang, B. Coord. Chem. Rev. 2006, 250, 242. (c) Xi, Q.; Zhang, W.; Zhang, X. Synlett 2006, 945. (d) Barberá, J.; Rakitin, O. A.; Ros, M. B.; Torroba, T. Angew. Chem. Int. Ed. 1998, 37, 296.

(e) Banide, E. V.; O'Connor, C.; Fortune, N.; Ortin, Y.; Milosevic, S.; Müller-Bunz, H.; McGlinchey, M. J. Org Biomol. Chem. 2010, 8, 3997. (f) Zargarian, D. Coord. Chem. Rev. 2002, 233-234, 157. (g) Morinaka, K.; Ubukata, T.; Yokoyama, Y. Org. Lett. 2009, 11, 3890. (h) Yang, J.; Lakshmikantham, M. V.; Cava, M. P.; Lorcy, D.; Bethelot, J. R. J. Org. Chem. 2000, 65, 6739. (i) Alt, H. G.; Köppl, A. Chem. Rev. 2000, 100, 1205.
(3) (a) Zhou, X.; Zhang, H.; Xie, X.; Li, Y.J. Org. Chem. 2008, 73, 3958. (b) Marion, N.; Díez-González, S.; de Frémont, P.; Noble, A. R.; Nolan, S. P. Angew. Chem. Int. Ed. 2006, 45, 3647. (c) Kurouchi, H.; Sugimoto, H.; Otani, Y.; Ohwada, T. J. Am. Chem. Soc. 2010, 132, 807. (d) Womack, G. B.; Angeles, J. G.; Fanelli, V. E.; Heyer, C. A. J. Org. Chem. 2007, 72, 7046. (e) Zhu, Z.-B.; Shi, M. Chem. Eur. J. 2008, 14, 10219. (f) Zhang, X.-M.; Tu, Y.-Q.; Jiang, Y.-J.; Zhang, Y.-Q.; Fan, C.-A.; Zhang, F.-M. Chem. Commun. 2009, 4726. (g) Li, C.; Zeng, Y.; Wang, J. Tetrahedron Lett. 2009, 50, 295. (h) Khan, Z. A.; Wirth, T. Org. Lett. 2009, 11, 229. (i) Zhang, D.; Yum, E. K.; Liu, Z.; Larock, R. C. Org. Lett. 2005, 7, 4963. (j) Bi, H.-P.; Liu, X.-Y.; Gou, F.-R.; Guo, L.N.; Duan, X.-H.; Liang, Y.-M. Org. Lett. 2007, 9, 3527. (k) Bryan, C. S.; Lautens, M. Org. Lett. 2010, 12, 2754. (1) Rayabarapu, D. K.; Cheng, C.-H. Chem. Commun. 2002, 9, 942. (m) Deng, R.; Sun, L.; Li, Z. Org. Lett. 2007, 9, 5207. (n) Kuninobu, Y.; Kawata, A.; Takai, K. J. Am. Chem. Soc. 2005, 127, 13498. (o) Miyamoto, M.; Harada, Y.; Tobisu, M.; Chatani, N. Org. Lett. 2008, 10, 2975. (p) Kuninobu, Y.; Tokunaga, Y.; Kawata, A.; Takai, K. J. Am. Chem. Soc. 2006, 128, 202. (q) Chang, K.-J.; Rayabarapu, D. K.; Cheng, C.-H. J. Org. Chem. 2004, 69, 4781.

(4) (a) Bu, X.; Hong, J.; Zhou, X. Adv. Synth. Catal. 2011, 353, 2111. (b) Liu, C.-R.; Yang, F.-L.; Jin, Y.-Z.; Ma, X.-T.; 
Cheng, D.-J.; Li, N.; Tian, S.-K. Org. Lett. 2010, 12, 3832. (c) Li, H.; Li, W.; Liu, W.; He, Z.; Li, Z. Angew. Chem. Int. Ed. 2011, 50, 2975.

(5) (a) Rong, Y.; Li, R.; Lu, W. Organometallics 2007, 26, 4376. (b) Li, Z.; Cao, L.; Li, C.-J. Angew. Chem. Int. Ed. 2007, 46, 6505. (c) Li, Y.-Z.; Li, B.-J.; Lu, X.-Y.; Lin, S.; Shi, Z.-J. Angew. Chem. Int. Ed. 2009, 48, 3817. (d) Correia, C. A.; Li, C.-J. Adv. Synth. Catal. 2010, 352, 1446.

(6) (a) Liu, X.; Zhang, Y.; Wang, L.; Fu, H.; Jiang, Y.; Zhao, Y. J. Org. Chem. 2008, 73, 6207. (b) Pelletier, G.; Powell, D. A. Org. Lett. 2006, 8, 6031. (c) Powell, D. A.; Fan, H. J. Org. Chem. 2010, 75, 2726. (d) Ye, Y.-H.; Zhang, J.; Wang, G.; Chen, S.-Y.; Yu, X. Q. Tetrahedron 2011, 67, 4649. (e) Wang, Z.; Zhang, Y.; Fu, H.; Jiang, Y.; Zhao, Y. Org. Lett. 2008, 10, 1863. (f) Xia, Q.; Chen, W.; Qiu, H. J. Org. Chem. 2011, 76, 7577.

(7) Qin, C.; Zhou, W.; Chen, F.; Ou, Y.; Jiao, N. Angew. Chem. Int. Ed. 2011, 50, 12595.

(8) (a) Rybtchinski, B.; Milstein, D. Angew. Chem. Int. Ed. 1999, 38, 870. (b) Li, Z.; Li, C.-J. J. Am. Chem. Soc. 2005, 127, 6968. (c) Coperet, C. Chem. Rev. 2010, 110, 656. (d) Sun, C.-L.; Li, B.-J.; Shi, Z.-J. Chem. Rev. 2011, 111, 1293. (e) Ramesh, D.; Ramulu, U.; Rajaram, S.; Prabhakar, P.; Venkateswarlu, Y. Tetrahedron Lett. 2010, 51, 4898. (f) Li, Z.; Yu, R.; Li, H. Angew. Chem. Int. Ed. 2008, 47, 7497. (g) Li, C.-J. Acc. Chem. Res. 2009, 42, 335.

(9) (a) Bolm, C.; Legros, J.; Le Paih, J.; Zani, L. Chem. Rev. 2004, 104, 6217. (b) Bolm, C. Nat. Chem. 2009, 1, 420. (c) Enthaler, S.; Junge, K.; Beller, M. Angew. Chem. Int. Ed.
2008, 47, 3317. (d) Fürstner, A.; Leitner, A.; Méndez, M.; Krause, H. J. Am. Chem. Soc. 2002, 124, 13856. (e) Sherry, B. D.; Fürstner, A. Acc. Chem. Res. 2008, 41, 1500. (f) Bauer, E. B. Curr. Org. Chem. 2008, 12, 1341.

(10) (a) Stang, P. J.; Anderson, A. G. J. Am. Chem. Soc. 1978, 100, 1520. (b) Viswanathan, G. S.; Wang, M. W.; Li, C. J. Angew. Chem. Int. Ed. 2002, 41, 2138. (c) Stang, P. J.; Rappoport, Z.; Hanack, M.; Subramanian, L. R. Vinyl Cations; Academic Press: New York, 1979.

(11) General Procedure for the Iron-Catalyzed Domino Reaction - Synthesis of 6-Benzhydryl-1,2,3-triphenyl$1 \mathrm{H}$-indene (3aa)

Diphenylacetylene 1a (44.5 mg, $0.25 \mathrm{mmol}), \mathrm{FeCl}_{2}(3.1 \mathrm{mg}$, $10 \mathrm{mmol} \%$ ), and NBS (97.9 mg, $0.55 \mathrm{mmol}$ ) were added to a flask with a magnetic stirring bar. The tube was evacuated and refilled with $\mathrm{N}_{2}$, and then diphenylmethane (2a, $210 \mu \mathrm{L}$, $1.25 \mathrm{mmol})$ and DCE $(2 \mathrm{~mL})$ was added. The resulting mixture was stirred at $80^{\circ} \mathrm{C}$ for $10 \mathrm{~h}$. After cooling to r.t., the mixture was diluted with EtOAc and filtered. The filtrate was removed under reduced pressure to get the crude product, which was further purified by silica gel chromatography (PE as eluent) to give product 3aa $(75 \%$ yield); white solid; mp 155-157 ${ }^{\circ} \mathrm{C} .{ }^{1} \mathrm{H}$ NMR $(300 \mathrm{MHz}$, $\left.\mathrm{CDCl}_{3}\right): \delta=7.40-7.28(\mathrm{~m}, 5 \mathrm{H}), 7.22-7.13(\mathrm{~m}, 8 \mathrm{H}), 7.10$ $6.98(\mathrm{~m}, 14 \mathrm{H}), 6.98-6.94(\mathrm{~m}, 1 \mathrm{H}), 5.50(\mathrm{~s}, 1 \mathrm{H}), 5.03(\mathrm{~s}, 1$ H). $\left.{ }^{13} \mathrm{C} \mathrm{NMR} \mathrm{(75} \mathrm{MHz,} \mathrm{CDCl}_{3}\right): \delta=148.4,145.5,144.1$, 144.0, 143.4, 141.6, 140.4, 139.7, 135.5, 129.4, 129.2, $129.1,128.6,128.5,128.2,128.1,127.8,127.4,126.5$, $126.1,125.3,120.1,57.9,56.8$. 